\title{
Study of resistance pattern of isolated micro-organisms towards commonly used anti-microbial agents in medical intensive care unit of a tertiary care teaching hospital
}

\author{
Mansij Biswas $^{1 *}$, Nirmala N. Rege ${ }^{1}$, Niteen D. Karnik ${ }^{2}$, Gita Nataraj ${ }^{3}$
}

\begin{abstract}
${ }^{1}$ Department of Pharmacology and Therapeutics, ${ }^{2}$ Department of Medicine, ${ }^{3}$ Department of Microbiology, Seth GS Medical College \& KEM Hospital, Mumbai, Maharashtra, India
\end{abstract}

Received: 24 October 2019

Revised: 08 December 2019

Accepted: 09 December 2019

\section{*Correspondence:}

Dr. Mansij Biswas,

Email: doctor.mansij@gmail.com

Copyright: ( $)$ the author(s), publisher and licensee Medip Academy. This is an open-access article distributed under the terms of the Creative Commons Attribution Non-Commercial License, which permits unrestricted non-commercial use, distribution, and reproduction in any medium, provided the original work is properly cited.

\begin{abstract}
Background: The objective of the study was to find out different types of biological samples from admitted patients tested for culture and sensitivity $(\mathrm{C} \& \mathrm{~S})$, prevalence of different types of organisms isolated from those samples, and to analyze the resistance pattern of those isolated organisms against commonly used or tested anti-microbial agents (AMAs).

Methods: Following institutional ethics committee approval and written informed consent, adult patients of both genders, receiving AMAs were enrolled from June 2014 to July 2015 and followed up daily till they were in medical intensive care unit (MICU). Demographic data, diagnosis, culture-sensitivity (antibiogram) and other investigation reports and treatment details were recorded. Descriptive statistical analysis of collected data was done.

Results: Of the 514 samples (from 600 patients enrolled) sent for C\&S testing, 143 were reported as sterile while from the rest 371 samples, 504 organisms were isolated; commonly isolated organisms were Pseudomonas aeruginosa (30\%), Acinetobacter baumannii (23\%), Klebsiella pneumoniae (16\%), Providencia sp. (7.1\%), Escherichia coli (5.7\%), and Enterobacter sp. (4.2\%). Samples were sent in $63 \%$ of enrolled patients, the commonest being broncho-alveolar lavage (48\% of total). Microbial resistance was high for cephalosporins (ceftriaxone, cefepime, ceftazidime), carbapenems (meropenem, imipenem), penicillins (piperacillin), quinolones (ciprofloxacin, levofloxacin), aminoglycosides (gentamicin, netilmicin, amikacin) and cotrimoxazole. Most organisms were sensitive to colistin (100\%), polymyxin B (92\%) and tigecycline $(69 \%)$.

Conclusions: The information regarding commonly isolated organisms and their resistant pattern would aid in rational selection of AMAs and thus the present study is useful to clinicians managing MICU and the hospital infection committee to plan future policies regarding AMA use in MICU.
\end{abstract}

Keywords: Multi-drug resistance, Superbugs, Antibiotic stewardship, Rational use of antibiotics, Antimicrobial resistance

\section{INTRODUCTION}

Intensive care units (ICU) cater to critically ill patients, who often suffer from several infections and have various co-morbid conditions associated with one or more existing or impending organ dysfunction. These patients generally receive multiple medications from different classes (polypharmacy). ${ }^{1,2}$ They also have potentially altered pharmacokinetics and pharmacodynamics of the administered drugs, mostly due to compromised hepatic or renal function. ${ }^{3}$ So, drug therapy of these patients is complicated and irrational use may expose a substantial 
number of them to drug resistance, interactions and adverse reactions leading to treatment failure. ${ }^{2}$

Antimicrobial agents (AMAs) are one of the most frequently prescribed classes of drug in a critical care setup both for therapy and prophylaxis, especially to tackle nosocomial infections in patients or to prevent in those who are at a higher risk of developing them (e.g. rheumatic fever, recurrent cellulitis, meningococcal disease, recurrent urinary tract infections, spontaneous bacterial peritonitis, infective endocarditis, acute necrotizing enterocolitis etc.). ${ }^{4,5}$ Extensive use of AMAs lead to increased drug resistance in such vulnerable group of patients. Thus their use may lower the risk of specific infections but at the cost of emergence of multi-drug resistant organisms. This contributes significantly towards the rise in escalating health care costs and patient morbidity and mortality, ultimately resulting in poor treatment outcome. ${ }^{6-8}$

Therefore, monitoring and evaluation of prescribing pattern of AMAs in critical care set up as well as maintaining records of pattern of prevailing organisms and their resistance towards different antibiotics are some of the recommended strategies to control widespread antibiotic resistance. Regular conduct of antibiotic stewardship programs is also helpful to arrive at a suitable guideline or policy to be followed at a particular setup. Realizing the need for antibiotic usage guidelines, the Global Antibiotic Resistance Partnership (GARP) was launched in order to develop actionable policy recommendations especially suited for developing nations. ${ }^{9-11}$ Subsequently, Indian Council of Medical Research (ICMR) has come up with standard treatment guidelines for antimicrobial use in several common indications for both therapy and prophylaxis including those in critical care setup and surgical site infections. ${ }^{12}$

With this background, it appeared logical and essential that any ICU setup should have a database of commonly isolated organisms from biological samples of admitted patients and their resistance pattern towards various AMAs. Though the medical ICU (MICU) of our institution, which is a tertiary care teaching hospital, caters to approximately 55-60 patients per month, yet such data has not been available in recent times. Hence, as the first step towards creating a database and establishing an appropriate anti-microbial usage policy, the present study was carried out. The objectives were to find out the prevalence of different organisms isolated from various biological samples subjected to culture \& sensitivity (C\&S) testing; and to analyze the resistance pattern of those isolated organisms against commonly used AMAs.

\section{METHODS}

\section{Study design and site}

A single center prospective observational study was carried out in the MICU of King Edward Memorial
(KEM) Hospital, Mumbai, a tertiary care teaching hospital.

\section{Ethics}

The study was initiated after obtaining permission from the institutional ethics committee (EC/49/2014) of Seth GS Medical College and KEM Hospital, Mumbai; and the procedures followed were in accordance with the ethical standards of the responsible committee on human experimentation (Indian Council of Medical Research, Ethical Guidelines for Biomedical Research on Human Participants, 2006) and with the Helsinki Declaration of 1975 , as revised in 2000 .

\section{Selection criteria}

Adult patients more than 18 years of age and of either gender admitted in MICU and receiving one or more AMAs as part of their treatment were included. Any patient or legally accepted representative (LAR) who refused to consent or re-consent after recovery, were excluded.

\section{Sample size}

Considering the annual bed occupancy rate of MICU, approval to enrol up to 600 patients over a period of approximately 14 months (June 2014 to July 2015) was sought and obtained from ethics committee. A duration specific convenience sampling method was adopted.

\section{Data collection and key variables recorded}

After obtaining written informed consent from the patient or the LAR and impartial witness, a baseline demographic data, history and clinical examination findings, working diagnosis, any co-morbidity and prescribed AMAs were recorded from the enrolled participant's file. Subsequent visits were made at least once daily, till the patient was admitted in MICU. During these visits, all the reports bearing the information of specimen sample sent, isolated organisms and antibiogram pertaining to each isolated organism were obtained, whenever done and available. These included any repeat testing of the same or different sample from one single patient as well. All specimens were processed as per standard laboratory practices for isolation of organisms and antimicrobial susceptibility tests were performed and interpreted as per Clinical and Laboratory Standards Institute (CLSI) standards. Any change in the antimicrobial therapy following availability of culture and sensitivity report was noted down. An appropriately designed case record form (CRF) was used to record all the data.

\section{Outcome measures}

Demographics, proportions of different biological samples sent for C\&S, proportions of different organisms 
isolated from those samples, extent of microbial resistance against commonly used AMAs, resistance pattern of commonly isolated individual organisms.

\section{Statistical analysis}

Descriptive statistical analysis of different variables was done using SPSS v21 (IBM Corp. Released 2012. IBM SPSS Statistics for Windows, Version 21.0. Armonk, NY: USA.), and the analyzed data were presented in numbers, percentages, tables and figures. Quantitative data were presented using measures of central tendency (median [range]). Categorical data were expressed as proportions.

\section{RESULTS}

\section{Demographics}

A total of 600 patients (286 male and 314 female) were enrolled during the study period of 14 months from June 2014 to July 2015. The median age of the enrolled participants was 30 years (range 18-86 years). Maximum number of patients $(n=375 ; 62.5 \%)$ were in the age group category of 18-35 years. This has been elaborated in Table 1. Of the 600 enrolled patients, 254 patients succumbed to the illness in MICU (mortality: 42.3\%); the rest either got discharged $(n=75 ; 12.5 \%)$, took discharge against medical advice $(n=20 ; 3.3 \%)$ or were transferred to general wards for further treatment $(n=251 ; 41.9 \%)$. The median duration of MICU stay was 5 days (range: 0-
385 days). Most of the patients $(n=461 ; 77 \%)$ had an ICU stay between one to 15 days. Thirty-seven $(6 \%)$ patients had a stay of 'zero' days i.e. they were transferred, discharged or succumbed to illness on the same day of MICU admission.

Table 1: Demographics of enrolled patients $(n=600)$.

\begin{tabular}{|lll|}
\hline Age group (years) & No. of patients & Gender (M:F) \\
\hline $\mathbf{1 8 - 3 5}$ & 375 & $166: 209$ \\
\hline $\mathbf{3 6 - 5 0}$ & 126 & $65: 61$ \\
\hline $\mathbf{5 1 - 6 5}$ & 77 & $46: 31$ \\
\hline $\mathbf{> 6 5}$ & 22 & $9: 13$ \\
\hline Total & 600 & $286: 314$ \\
\hline
\end{tabular}

Note: M-Male, F-Female.

\section{Samples for $C \& S$}

Different biological samples of $378 \quad(63 \%$ of total enrolled) patients were subjected to C\&S testing. Total 514 samples were sent from these patients (average 1.36 per patient). Of the samples tested, $143(27.8 \%)$ revealed no organism on aerobic culture (sterile). Rest 371 samples yielded a total of 504 organisms. These have been depicted in Figure 1. Broncho-alveolar lavage was the most commonly sent specimen $(\mathrm{n}=247 ; 48 \%)$, followed by blood $(n=126 ; 24.5 \%)$, Urine $(n=58 ; 11.3 \%)$ and Central line tip $(n=40 ; 7.8 \%)$. This has been elaborated in Table 2. In $87(23 . \%)$ of 378 patients for whom samples were sent for $\mathrm{C} \& \mathrm{~S}$ testing, the already existing AMA regimen was changed according to the $C \& S$ pattern.

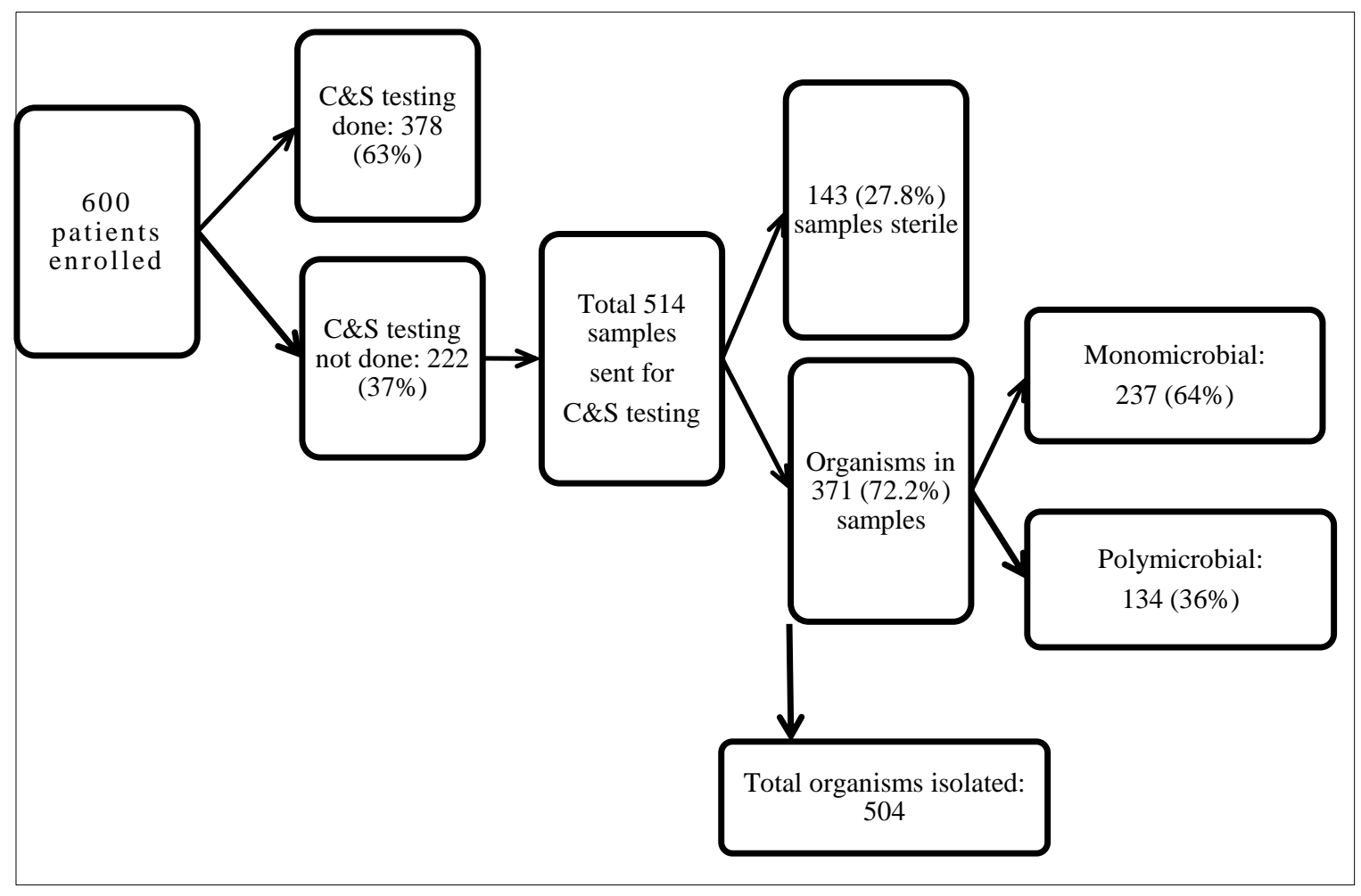

Figure 1: Culture and sensitivity testing. 


\section{Organisms isolated}

Sixty four percent of those culture positive samples were monomicrobial $(\mathrm{n}=237)$, while rest $36 \%$ were polymicrobial $(n=134)$. Among the blood samples, $74 \%$
(93 of 126) were sterile (Table 2). Pseudomonas aeruginosa (152 of 504, 30\%) was the most commonly isolated organism followed by Acinetobacter baumannii (117 of 504, 23\%) and Klebsiella pneumoniae (82 of 504, $16 \%$ ). This has been elaborated in Table 3.

Table 2: Types of samples sent for C\&S (n=514).

\begin{tabular}{|lllll|}
\hline Sample & Total $(\%)$ & Sterile & Mono microbial & Poly microbial \\
\hline Broncho-alveolar lavage & $247(48.2)$ & 12 & 123 & 112 \\
\hline Blood & $126(24.6)$ & 93 & 31 & 2 \\
\hline Urine & $58(11.3)$ & 10 & 37 & 11 \\
\hline Central line tip & $40(7.8)$ & 13 & 23 & 4 \\
\hline Sputum & $8(1.5)$ & 0 & 6 & 0 \\
\hline High vaginal swab & $7(1.3)$ & 0 & 7 & 3 \\
\hline Bed sore swab & $6(1.2)$ & 0 & 3 & 0 \\
\hline Synovial fluid & $6(1.2)$ & 3 & 3 & 0 \\
\hline Throat swab & $4(0.7)$ & 0 & 4 & 0 \\
\hline Ascitic fluid & $3(0.6)$ & 3 & 0 & 0 \\
\hline Bleb fluid & $3(0.6)$ & 3 & 0 & 0 \\
\hline Cerebro-spinal fluid & $3(0.6)$ & 3 & 0 & 0 \\
\hline Peritoneal fluid & $3(0.6)$ & 3 & 0 & \\
\hline
\end{tabular}

Table 3: Different organisms isolated from samples $(n=504)$.

\begin{tabular}{|llllll|}
\hline Organisms isolated & Frequency $(\%)$ & BAL & Blood & Urine & Others \\
\hline Pseudomonas aeruginosa & $152(30.2)$ & 125 & 4 & 11 & 12 \\
\hline Acinetobacter baumannii & $117(23.2)$ & 105 & 3 & 0 & 9 \\
\hline Klebsiella pneumoniae & $82(16.3)$ & 48 & 12 & 5 & 17 \\
\hline Providencia sp. & $36(7.1)$ & 13 & 0 & 20 & 3 \\
\hline Escherichia coli & $29(5.6)$ & 11 & 0 & 14 & 4 \\
\hline Enterobacter sp. & $21(4.2)$ & 21 & 0 & 0 & 0 \\
\hline Non fermenter Gram-negative bacillus & $17(3.4)$ & 14 & 0 & 0 & 3 \\
\hline Enterococcus sp. & $6(1.2)$ & 0 & 0 & 6 & 0 \\
\hline $\begin{array}{l}\text { Methicillin resistant coagulase negative } \\
\text { Staphylococcus }\end{array}$ & $6(1.2)$ & 0 & 3 & 0 & 3 \\
\hline Morganella morganii & $4(0.8)$ & 4 & 0 & 0 & 0 \\
\hline Methicillin resistant Staphylococcus aureus & $4(0.8)$ & 4 & 0 & 0 & 0 \\
\hline $\begin{array}{l}\text { Methicillin resistant } \\
\text { Staphylococcus } \text { hemolyticus }\end{array}$ & $4(0.8)$ & 0 & 0 & 0 & 4 \\
\hline Burkholderrhoea species & $4(0.8)$ & 0 & 4 & 0 \\
\hline Sphingomonas paucinobilis & $4(0.8)$ & 0 & 4 & 0 \\
\hline Candida albicans & $3(0.6)$ & 0 & 0 & 0 \\
\hline Citrobacter sp. & $3(0.6)$ & 0 & 0 & 3 \\
\hline Gram-negative bacilli (non specific) & $3(0.6)$ & 0 & 0 & 0 \\
\hline Gram-negative cocci (non specific) & $3(0.6)$ & 0 & 0 & 0 \\
\hline Non-albicans Candida & $3(0.6)$ & 0 & 3 & 0 \\
\hline Proteus sp. & $3(0.6)$ & 3 & 0 & 0 \\
\hline
\end{tabular}

\section{Microbial resistance against commonly used AMAs}

Microbial resistance was high against cephalosporins (89\% organisms resistant to ceftriaxone, $78 \%$ to cefepime, $78 \%$ to ceftazidime), carbapenems (83\% organisms resistant to meropenem, $49 \%$ to imipenem), penicillins ( $71 \%$ organisms resistant to piperacillin+tazobactam), quinolones ( $85 \%$ organisms resistant to ciprofloxacin, $78 \%$ to levofloxacin), aminoglycosides (83\% organisms resistant to gentamicin, $79 \%$ to netilmicin, $77 \%$ to amikacin) and cotrimoxazole (92\% organisms resistant). Most of the organisms were sensitive to colistin (100\%), polymyxin B $(92 \%)$ and tigecycline $(69 \%)$. This has been elaborated in Table 4. 
Table 4: Percentage of resistance profile of all organisms against various commonly used or tested AMAs.

\begin{tabular}{|c|c|c|c|c|}
\hline AMAs & No. of samples & Resistant & Sensitive & $\%$ Resistance \\
\hline Cotrimoxazole & 242 & 223 & 19 & 92 \\
\hline Ceftriaxone & 331 & 295 & 36 & 89 \\
\hline Ciprofloxacin & 293 & 248 & 45 & 85 \\
\hline Gentamicin & 415 & 343 & 72 & 83 \\
\hline Meropenem & 261 & 216 & 45 & 83 \\
\hline Netilmicin & 179 & 142 & 37 & 79 \\
\hline Cefepime & 109 & 85 & 24 & 78 \\
\hline Ceftazidime & 449 & 349 & 100 & 78 \\
\hline Levofloxacin & 300 & 235 & 65 & 78 \\
\hline Amikacin & 394 & 302 & 92 & 77 \\
\hline Piperacillin and Tazobactam & 282 & 200 & 82 & 71 \\
\hline Imipenem & 329 & 163 & 166 & 49 \\
\hline Tigecycline & 177 & 55 & 122 & 31 \\
\hline Polymyxin B & 258 & 20 & 238 & 8 \\
\hline Colistin & 103 & 0 & 103 & 0 \\
\hline
\end{tabular}

Table 5: Resistance pattern of Pseudomonas aeruginosa against commonly tested AMAs.

\begin{tabular}{|llll|}
\hline AMAs & \% resistance & AMAs & \% resistance \\
\hline Ceftazidime & 62 & Pip+Taz & 63 \\
\hline Gentamicin & 86 & Meropenem & 86 \\
\hline Amikacin & 74 & Cotrimoxazole & 100 \\
\hline Ceftriaxone & 100 & Netilmicin & 62 \\
\hline Imipenem & 58 & Tigecycline & 100 \\
\hline Levofloxacin & 80 & Cefepime & 70 \\
\hline Ciprofloxacin & 86 & PolymyxinB/Colistin & 0 \\
\hline
\end{tabular}

Table 6: Resistance pattern of Acinetobacter baumannii against commonly tested AMAs.

\begin{tabular}{|llll|}
\hline AMAs & \% resistance & AMAs & \% resistance \\
\hline Gentamicin & 88 & Meropenem & 87 \\
\hline Amikacin & 92 & Cotrimoxazole & 100 \\
\hline Ceftriaxone & 92 & Netilmicin & 100 \\
\hline Imipenem & 67 & Tigecycline & 22 \\
\hline Levofloxacin & 97 & Cefepime & 100 \\
\hline Ciprofloxacin & 91 & PolymyxinB/Colistin & 0 \\
\hline
\end{tabular}

Table 7: Resistance pattern of Klebsiella pneumoniae against commonly tested AMAs.

\begin{tabular}{|llll|}
\hline AMAs & \% resistance & AMAs & \% resistance \\
\hline Ceftazidime & 100 & Pip+Taz & 87 \\
\hline Gentamicin & 75 & Meropenem & 75 \\
\hline Amikacin & 61 & Cotrimoxazole & 86 \\
\hline Ceftriaxone & 89 & Netilmicin & 89 \\
\hline Imipenem & 50 & Tigecycline & 0 \\
\hline Levofloxacin & 64 & Cefepime & 100 \\
\hline Ciprofloxacin & 82 & Polymyxin B/colistin & 0 \\
\hline
\end{tabular}

\section{Resistance pattern of individual organisms}

$P$. aeruginosa and $A$. baumannii expressed high level of resistance against most of these AMAs except colistin and polymyxin $\mathrm{B}$, for which they were $100 \%$ sensitive. $K$. pneumoniae expressed similar pattern, but in addition to colistin and polymyxin B, it was also found to be $100 \%$ sensitive to tigecycline. Providencia sp. was highly sensitive to imipenem with cilastatin and tigecycline but found to be resistant to polymyxin B. Sensitivity of 
Providencia sp. to colistin was not tested. Escherichia coli was found to be sensitive to imipenem+cilastatin, amikacin, levofloxacin, tigecycline, polymyxin B and colistin. Enterobacter sp. was sensitive to imipenem and cilastatin, piperacillin and tazobactam, tigecycline and polymyxin B. Both Providencia sp. and E. coli were highly resistant towards other AMAs. These have been elaborated in Tables 5-7.

\section{DISCUSSION}

It was a common practice to send BAL fluid for C\&S testing from intubated patients, accounting for the high frequency noted for BAL sent as specimen sample. Bronchoalveolar lavage (BAL) and bronchial wash (BW) are common flexible bronchoscopic techniques performed routinely in the intensive care units to obtain samples of airway secretions for microbiological, cytological or immunological analysis. ${ }^{13}$ Of the total samples sent for C\&S testing, only $28 \%$ were reported as sterile, reflecting high incidence of culture positive samples from the admitted patients. Among them, $2 / 3^{\text {rd }}$ was monomicrobial (64\%). Wattal, et al reported similar results in their study with $67.5 \%$ monomicrobial samples, though they recorded only blood culture samples and only $12.7 \%$ of the total samples tested to be positive for any organism. ${ }^{14}$ Our study revealed contrasting high rate of culture positivity probably because we tested all types of body samples including BAL and urine, which yield higher positivity than blood. Interestingly, in our study also only $26 \%$ of the blood samples showed culture positivity. In less than one-fourth of the patients (only 23\%) for whom samples were sent for $\mathrm{C} \& \mathrm{~S}$ testing, the already existing AMA regimen was changed according to the sensitivity pattern. This was mostly due to the fact that treating physicians were keener to rely upon clinical response of the patients on the existing AMA regimen, rather than merely depending upon the microbiological results.

High percentage of $P$. aeruginosa (30\%), A. baumannii $(23 \%)$ and $K$. pneumoniae $(16 \%)$ was detected in these samples with a very low percentage of Candida sp. $(1.2 \%)$. A study in North India by Sharma, et al found that the most common organism isolated was Acinetobacter (26.1\%) followed by Candida $(23.8 \%)$ and Pseudomonas $(21.4 \%) .{ }^{15}$ Wattal, et al found coagulase negative staphylococcus (CoNS) as the commonest single isolate among ICU patients (20.3\%) followed by Candida sp. (17.5\%), Klebsiella sp. (15.7\%) and Acinetobacter sp. $(12 \%) .{ }^{14}$ But the fact to consider here is CoNS isolated from blood cultures are often contaminants. Difference observed in Candida isolation from our ICU with other ICUs in India might be related to the geographical locations, disease profile of patients and facilities available for fungal culture. A recent review article on antimicrobial resistance in intensive care unit states that the most important resistant microorganisms in the ICU setup are methicillin-resistant Staphylococcus aureus (MRSA, among Gram-positive organisms), extendedspectrum beta-lactamase (ESBL) producers of Klebsiella sp. and Escherichia coli (among Gram-negative organisms); and multidrug resistant $P$. aeruginosa, Acinetobacter species and Enterobacter sp. ${ }^{16}$ The pattern of resistant organisms observed in our set up was mostly similar except that frequency of MRSA isolates was very less.

The organisms isolated from the samples indicate that a therapy was warranted with third generation cephalosporins such as ceftriaxone and cefoperazone or extended spectrum penicillins (for P. aeruginosa, E. coli, $K$. pneumonia), carbapenems (for $P$. aeruginosa, $A$. baumannii), aminoglycosides preferably in combination with a beta lactam antibiotic (for $P$. aeruginosa, $K$. pneumonia, Providencia sp.), quinolones such as levofloxacin (for E. coli, Enterobacter sp., $P$. aeruginosa). However, when the susceptibility to these organisms was tested in in vitro they were found to be highly resistant. Thus, it appears that these so called 'higher' AMAs which have been introduced only in the last two decades viz. meropenem or levofloxacin (both in 1996) are losing their efficacy against MDR organisms isolated from MICU patients. ${ }^{17,18}$ It is well known that mortality, morbidity, and health care costs are substantially increased by infections caused by such MDR organisms. ${ }^{19}$ Present study also showed high mortality among admitted patients.

Linezolid was introduced in 2001 and shown to be active against $>98 \%$ of Staphylococcus, with resistance identified in $0.05 \%$ of $S$. aureus and $1.4 \%$ of coagulasenegative Staphylococcus. ${ }^{20}$ In the present study testing for linezolid sensitivity was done in only 17 samples and all the organisms (Methicillin resistant Staphylococci, Coagulase negative Staphylococci, Enterococci), showed sensitivity towards it. But the number of samples were too less to derive any valid conclusion regarding resistance.

Our results also indicate that the isolated organisms (such as $A$. baumannii and $K$. pneumonia) showed relatively better sensitivity $(69 \%)$ against tigecycline. The latter was developed and given a fast-track approval by the U.S. Food and Drug Administration (FDA) in 2005 for the treatment of complicated skin and soft tissue infections, intra-abdominal infections and community-acquired pneumonia. $^{21}$ Despite this, more than $30 \%$ organisms were found to be resistant to tigecycline, indicating a slowly rising resistance for this new AMA as well, $P$. aeruginosa being the main culprit with complete (100\%) resistance.

On the other hand, almost all organisms were sensitive to colistin and polymyxin B; exception being Providencia sp. being resistant against polymyxin $\mathrm{B}$, which is expected because of the inherent resistance of the organism. Of the polymyxins, only polymyxin B and polymyxin $\mathrm{E}$ (colistin) have been introduced in clinical practice in 1949. However due to safety issues like nephrotoxicity, ototoxicity, and neuromuscular blockade, they got relegated to backbenches. They however gained 
clinical importance again in late 1990s as resurgence of MDR Gram-negative organisms has been on the rise. ${ }^{22,23}$

Many a times for a single patient during his/her stay in MICU, biological samples were sent for C\&S on multiple occasions. However, data pertaining to change in the AMA regimen following availability of C\&S reports was not analyzed in details, which may be considered as the limitation of the present study; as then it would have been possible to comment on the rationality of AMA prescribing. However, assessment of the rationality of AMA prescribing was not the objective of the study. Also, from the study, it was not possible to deduce the extent to which the mortality was related to infections by resistant organisms.

Thus the above-mentioned analysis of resistance pattern of the isolated organisms cautions us about drastically narrowed down therapeutic options against them and emphasizes judicious use of AMAs, as the future pipeline of newer AMA development is also not very promising. It also points out that our MICU team with Hospital infection control Committee and Antibiotic Policy Development Committee need to adopt measures like optimizing the duration of empirical treatment or by restricting AMA use to proven or probable infections only, guided by strict AMA policy and regular antibiotic stewardship programs, supported by confirmed microbiological reports. Rotation or cycling of AMAs can also be a measure to reduce selection pressure on behalf of the physicians. Continuous cycling of AMAs by removing single AMA or an entire class from the formulary for a certain period and re-introducing it/them later denies micro-organisms to develop resistance to any particular agent or class of agents to a great extent. At present, this method has gained considerable interest globally. Though earlier reports do not suggest routine use of this method, but a recent meta-analysis concluded that adjustable cycling with a cycling period of one month is especially useful to suppress emergence of multiple resistance, while too long cycling periods may be detrimental. $^{24-26}$ Another important consideration to be made here is that the epidemiology of infections in Indian ICUs is different from that of western countries in terms of source of infection, prevalence and nature of microorganisms causing it. While Gram-positive infections are more prevalent in ICUs of western countries, the Indian ICUs are more commonly infected with Gram-negative organisms showing a high degree of antimicrobial resistance, as depicted in our study as well. ${ }^{27}$ Therefore, blindly following the standard antimicrobial guidelines, which are mostly developed, based on research and evidences from western countries, may fail to provide the optimum results in India.

\section{CONCLUSION}

A hospital policy regarding AMAs available for use, through which a choice can be made taking into consideration the organisms' susceptibility should be in place. A database of commonly isolated organisms and their resistance pattern towards commonly used AMAs would be helpful in preventing emergence of multi-drug resistance. The information regarding the same would aid in rational selection of AMAs. Thus, the present study is useful to clinicians managing MICU and the Hospital Infection Committee to plan future policies regarding AMA use in MICU based on local/institutional epidemiological data and international guidelines. Implementation of these recommendations would strengthen the quality of patient care and benefit the entire health care system at large.

\section{ACKNOWLEDGEMENTS}

Authors would like to thank the MICU staff and all the patients.

\section{Funding: No funding sources}

Conflict of interest: None declared

Ethical approval: The study was approved by the Institutional Ethics Committee of Seth GS Medical College \& KEM Hospital, Mumbai (EC/49/2014)

\section{REFERENCES}

1. John LJ, Devi P, John J, Guido S. Drug utilization study of antimicrobial agents in medical intensive care unit of a tertiary care hospital. Asian J Pharm Clin Res. 2011;4:81-4.

2. Smythe MA, Melendy S, Jahns B. An exploratory analysis of medication utilization in a medical intensive care unit. Crit Care Med. 1993;21(9):31923.

3. Lin S, Foster KJ, Debellis RJ, Smith BS. Drug dosing in renal and hepatic failure: a pharmacokinetic approach to the critically ill patient. In: Irwin RS, Rippe JM, editors. Irwin and Rippe's Intensive Care Medicine. 7th ed. Philadelphia: Lippincott-Raven Publishers; 2011: 893-916.

4. Granowitz EV, Brown RB. Adverse reactions to antibiotics. In: Cunha BA, editor. Infectious diseases in critical care medicine. 2nd ed. New York: Informa Healthcare USA, Inc.; 2007: 575-594.

5. Enzler MJ, Berbari E, Osmon DR. Antimicrobial Prophylaxis in Adults. Mayo Clin Proc. 2011;86(7):686-701.

6. Niederman MS. Appropriate use of antimicrobial agents: Challenges and strategies for improvement. Crit Care Med. 2003;3:608-16.

7. Niederman MS. Use of broad-spectrum antimicrobials for the treatment of pneumonia in seriously ill patients: maximizing clinical outcomes and minimizing selection of resistant organisms. Clin Infect Dis. 2006;42:72-81.

8. Pulcine C, Pradier C, Samat-Long C, Hyvernat H, Bernardin G, Ichai C. Factors associated with adherence to infectious diseases advice in two intensive care units. J Antimicrob Chemother. 2006;57:546-50. 
9. Banerjee T, Anupurba S, Singh DK. Poor compliance with the antibiotic policy in the intensive care unit of a tertiary care hospital in India. J Infect Dev Ctries. 2013;7(12):994-8.

10. Goldman DA, Weinstein RA, Wenzel RP. Strategies to prevent and control the emergence of antimicrobial resistant micro-organisms in hospital. JAMA. 1996;275:234-49.

11. Davey P, Brown E, Charani E, Fenelon L, Gould IM, Homes A, et al. Interventions to improve antibiotic prescribing practices for hospital inpatients. Cochrane Database of Syst Rev. 2013;4:CD003543.

12. Indian Council of Medical Research, Department of Health Research New Delhi, India. Treatment Guidelines for Antimicrobial use in Common Syndromes; 2017. Available at: http://www.icmr. nic.in/guidelines/treatment $\% 20$ guidelines $\% 20$ for $\% 20$ antimicrobial.pdf. Accessed on 20 October 2018.

13. Mcgrath B, Drake J. Bronchoscopy in the ICU: An overview of broncho-alveolar lavage and bronchial washing. 2014. Available at: http://www.airwayelearning.com/awel/articles/articles-1.aspx? Action=1\&NewsId $=2115 \& \mathrm{M}=\mathrm{NewsV} 2 \& \mathrm{PID}=7165$. Accessed on 20 October 2018.

14. Wattal C, Raveendran R, Goel N, Oberoi JK, Rao BK. Ecology of blood stream infection and antibiotic resistance in intensive care unit at a tertiary care hospital in North India. Braz $\mathbf{J}$ Infect Dis. 2014;18(3):245-51.

15. Sharma PR, Burman P. Antimicrobial consumption and impact of "Reserve antibiotic indent form" in an intensive care unit. Indian $\mathbf{J}$ Pharmacol. 2010;42(5):297-300.

16. Brusselaers N, Vogelaers D, Blot S. The rising problem of antimicrobial resistance in the intensive care unit. Ann Intensive Care. 2011;1:47.

17. Highlights Of Prescribing Information, MERREM® I.V. Available at: https://www.accessdata. fda.gov/drugsatfda_docs/label/2014/050706s035lbl.p df. Accessed on 20 October 2018.

18. Highlights Of Prescribing Information, LEVAQUIN@. Available at: https://www.accessdata. fda.gov/drugsatfda_docs/label/2013/020634s065,020 635s071,021721s032lbl.pdf. Accessed on 20 October 2018.

19. Sydnor ERM, Perl TM. Hospital Epidemiology and Infection Control in Acute-Care Settings. Clin Microbiol Rev. 2011;24:141-73.

20. Gu B, Kelesidis T, Tsiodras S, Hindler J, Humphries RM. The emerging problem of linezolid-resistant Staphylococcus. J Antimicrob Chemother. 2013;68:4-11.

21. Greer ND. Tigecycline (Tygacil): the first in the glycylcycline class of antibiotics. Proc (Bayl Univ Med Cent). 2006;19(2):155-61.

22. Gupta S, Govil D, Kakar PN, Prakash O, Arora D, Das S, et al. Colistin and polymyxin B: A reemergence. Indian J Crit Care Med. 2009;13(2):4953.

23. Karnik ND, Sridharan K, Jadhav SP, Kadam PP, Naidu RK, Namjoshi RD, et al. Pharmacokinetics of colistin in critically ill patients with multidrug-resistant Gram-negative bacilli infection. Eur J Clin Pharmacol. 2013;69(7):1429-36.

24. Brown EM, Nathwani D. Antibiotic cycling or rotation: a systematic review of the evidence of efficacy. J Antimicrob Chemother. 2005;55:6-9.

25. Bergstrom CT, Lo M, Lipsitch M. Ecological theory suggests that antimicrobial cycling will not reduce antimicrobial resistance in hospitals. Proc Nat Acad Sci. 2004;101(36):13285-90.

26. Wiesch PA, Kouyos R, Abel S, Viechtbauer W, Bonhoeffer S. Cycling Empirical Antibiotic Therapy in Hospitals: Meta-Analysis and Models. PLoS Pathog. 2014;10(6):e1004225.

27. Chaudhry D, Prajapat B. Intensive care unit bugs in India: How do they differ from the Western world? J Assoc Chest Physicians. 2017;5(1):10-7.

Cite this article as: Biswas M, Rege NN, Karnik ND, Nataraj G. Study of resistance pattern of isolated micro-organisms towards commonly used antimicrobial agents in medical intensive care unit of a tertiary care teaching hospital. Int $\mathrm{J}$ Basic Clin Pharmacol 2020;9:158-65. 